\title{
Revisão Sistemática das aplicações em Administração do uso dos Métodos de Decisão Multicritério nas organizações.
}

\author{
Augusto Sérgio da Silva Souza - augusto.sergio@ufpe.br ${ }^{1}$ \\ Taciana de Barros Jerônimo - taciana.jeronimo@ufpe.br ${ }^{1}$
}

\begin{abstract}
Resumo - A Revisão Sistemática da Literatura (RSL) consiste em investigar o conhecimento produzido em determinada área através de uma metodologia rigorosa e formal. Esta RSL tem como objetivo analisar a literatura publicada entre os anos 2016-2020 a respeito da aplicação de modelos de decisão multicritério como ferramenta de apoio na gestão organizacional. A aplicação dos procedimentos metodológicos descritos neste trabalho resultou no retorno de 1.787 estudos de quatro bases cientificas selecionadas para esta pesquisa. Desta quantia, 231 artigos foram selecionados para a fase de análise de dados. Foram encontrados diversos exemplos de estruturação racional de decisão em problemas em oito grupos diferentes de organizações.
\end{abstract}

Palavras-chave: revisão sistemática da literatura, tomada de decisão multicritério, administração, organizações.

\section{Systematic Review of applications in Management of the use of Multicriteria Decision Methods in organizations}

\begin{abstract}
The Systematic Literature Review (SLR) consists of investigating the knowledge produced in a given area through a rigorous and formal methodology. This SLR aims to analyze the literature published between the years 2016-2020 regarding the application of multicriteria decision models as a support tool in organizational management. The application of the methodological procedures described in this work resulted in the return of 1.787 studies from four scientific bases selected for this research. Of this amount, 231 articles were selected for the data analysis phase. Several examples of rational decision structuring in problems were found in eight different groups of organizations.
\end{abstract}

Keywords: systematic literature review, multicriterial decision making, business, organizations.

Data da Submissão: 27/01/2021

Data de aceitação: 11/03/2021

Agradecimentos: $O$ presente trabalho foi realizado com apoio da Coordenação de Aperfeiçoamento de Pessoal de Nível Superior - Brasil (CAPES) - Código de Financ. 001. 


\section{Introdução}

Os métodos de decisão multicritério são utilizados quando existem análises com múltiplas alternativas, organização de trade-off e a influência de critério e do estilo do decisor no momento de escolher, elencar, priorizar e ordenar qual o curso de ações que será adotado pela organização a fim de desenvolver, melhorar e implementar uma estratégia, como também pode ser aplicado para diminuir e eliminar um problema existente.

A importância do estudo dos modelos de tomada de decisão multicritério na administração está na possibilidade de elucidar mentalmente quais as escolhas mais racionais que o gestor pode fazer e assim, contribuir para a sustentabilidade organizacional, mercadológica e financeira de um negócio.

Deste modo, esta Revisão Sistemática da Literatura (RSL) tem como objetivo analisar a literatura existente sobre a aplicação de modelos de decisão multicritério como ferramenta de apoio na gestão organizacional. Esta pesquisa busca identificar como estes modelos foram aplicados em diferentes contextos do processo decisório e quais os principais cenários para a adoção de determinado método aplicado na administração das organizações, uma vez que, entende-se que administração é o processo de planejar, digerir, organizar, mensurar, controlar os recursos disponíveis (humanos, materiais e não materiais) que interagem com o meio externo para alcançar os objetivos organizacionais (LACOMBE, 2009).

A abordagem metodológica pretendida para o desenvolvimento deste estudo será a investigação nas principais bases de dados de periódicos que utilizam, sobretudo, a aplicação multicritério simples (sem combinação de diferentes métodos multicritério) em organizações independentemente porte e setor, a fim de compreender as características acerca da sua utilização e posterior agrupamento e categorização dos principais pontos pretendidos pelo estudo que serão descritos na metodologia.

A lista de métodos e setores de adoção dos modelos pode ser utilizada como base para futuras pesquisas empíricas sobre o tema, além de servir como um guia de adoção parar nortear processos de tomada de decisão que apresentarem similaridades aos contextos e características descritas e encontradas na literatura analisada.

\section{Decisão multicritério}

No ano de 1960, a "teoria da decisão" foi desenvolvida por Howard Raiffa e Robert Schlaifer, na Harvard University's Business School. Desde então, a teoria abstrata foi aprimorada em pesquisa operacional e utilizada em diversos cenários, objetos e problemas, para atingir possíveis consequências e/ou resultados. Atualmente, dos estudos a respeito do processo decisório, emergem métodos e metodologias direcionadas a solucionar problemas que envolvem múltiplos critérios. Dentro da abordagem multicritério há duas escolas principais, uma conhecida como MCDM (Multicriteria Decision Making), de origem americana e visão objetivista; e outra conhecida como MCDA (Multicriteria Decision Aid), de origem europeia e visão subjetivista (RAIFFA, 1977).

A decisão multicritério consiste em uma escolha dentre, pelo menos, duas alternativas que se deseja atender a múltiplos objetivos, às vezes conflitantes entre si, com avaliação compensatória ou não; de sobreclassificação ou não; além da utilização de escalas de valor para 
a análise dos critérios entre as alternativas. Nesta perspectiva, é oportuno evidenciar o que o decisor pretende obter como resposta ao modelo, se é uma resposta única ou melhor solução entre todas; se é um ranking das melhores até as piores alternativas; se é um portfólio de ideias, visto que cada problemática levará a um conjunto de métodos específicos para a modelagem matemática da situação enfrentada por uma organização (JERÔNIMO, 2013). Também são encontrados na literatura os métodos multicritério de apoio à decisão, eles são ferramentas racionais que permitem aos decisores avaliar um conjunto de alternativas, considerando múltiplos critérios, elucidando a estrutura de preferência do decisor, além de considerar o desempenho das alternativas em cada critério estabelecido (ALMOGHATHAWI et al., 2017).

Um critério, de forma geral, é uma representação de um objetivo (ALMEIDA, 1999), podendo ser definido, de maneira mais formal, através da função de aferição de desempenhos em determinado objetivo especificado. É importante considerar que nos métodos multicritério, cada objetivo está associado a um critério, o que permitirá a avaliação das alternativas com base nestas metas e no estabelecimento das preferências do decisor.

Este enfoque visa apoiar o processo decisório por meio da recomendação de ações ou curso de ações, a fim de esclarecer o processo de formulação e implementação de estratégia. Na tentativa de incorporar os julgamentos e valores dos decisores, o processo de escolha de suas preferências é compreendido como um processo de aprendizagem, comunicação e discussão interativa. A preferência do decisor é influenciada por resultados sociais, neste sentido, a utilidade dos métodos é de esfera coletiva.

São encontradas na literatura diversas abordagens metodológicas de apoio à decisão multicritério, classificadas em três perspectivas conforme Guarnieri (2015), sendo elas abordagens do critério único de síntese; sobreclassificação ou subordinação e; métodos interativos. A escolha de um determinado método deve ser feita baseada no próprio problema e não o contrário. O Quadro 1 exemplifica métodos simples, ou seja, que não há combinação de mais de um tipo de método multicritério. Entretanto, esse rol não é exaustivo, existem mais abordagens que não constam nesta listagem.

Quadro 1 - Modelos e abordagens de apoio à decisão multicritério.

\begin{tabular}{|l|l|}
\hline \multicolumn{1}{|c|}{ MODELO } & \multicolumn{1}{c|}{ REFERÊNCIA } \\
\hline Abordagem Fuzzy & Bellman; Zadeh (1970) \\
\hline AHP & Saaty (1980) \\
\hline Análise Multivariada & Hair et al. (2009) \\
\hline ANP & Saaty (1996) \\
\hline Data Envelopment Analysis (DEA) & Charnes; Cooper; Rhodes (1978) \\
\hline ELECTRE e família & Roy (1968); Roy; Bertier (1971) \\
\hline Grey based approach & Deng (1989); Zhang; Wu; Olson (2005) \\
\hline MACBETH & Bana e Costa; Vansnick (1994) \\
\hline Mineração de dados & Tan; Steinbach; Kumar (2009) \\
\hline Multi-Attribute Utility Theory (MAUT) & Keeney; Raiffa (1976) \\
\hline Programação inteira & Vanderbei (1996) \\
\hline Programação multiobjetivo & Zenely (1974). \\
\hline PROMETHEE e família & $\begin{array}{l}\text { Brans; Vincke (1985); Brans; Vincke; Mareschal } \\
\text { (1986) }\end{array}$ \\
\hline
\end{tabular}




\begin{tabular}{|l|l|}
\hline Redes Neurais & Haykin (2001) \\
\hline Rough set Theory & Pawlak (1982); Slowinski; Greco; Matarazzo (2012) \\
\hline SMART & Edwards; Barron (1994) \\
\hline SMARTS & Edwards; Barron (1994) \\
\hline Theory of Constraints (TOC) & Simatupang; Wright; Sridharan (2004) \\
\hline TOPSIS & Hwang; Yoon (1981) \\
\hline
\end{tabular}

Em todos os tipos de métodos, as principais características encontradas são: cada ator tem sua subjetividade e deve ser respeitada; em processos decisórios complexos, há diferentes atores envolvidos que definem os aspectos relevantes que devem ser incorporados na modelagem matemática; reconhecem os limites da objetividade por meio da indicação dos pesos e da avaliação trade-off e; tem como pressuposto que o problema geralmente não está claramente definido (JERÔNIMO, 2013).

Em síntese, os benefícios da utilização da abordagem multicritério consistem na melhora da transparência do processo decisório; define, precisa e coloca em evidência a responsabilidade daquele que tem poder de decisão e; proporciona integridade, reprodutibilidade, objetividade e isonomia no tratamento de critérios.

\section{Revisão Sistemática da Literatura}

A Revisão Sistemática da Literatura (RSL) é uma metodologia rigorosa que estabelece, através de um processo formal e replicável, a investigação de uma questão de pesquisa ou fenômeno de interesse, cujos objetivos principais consistem em levantar o conhecimento produzido em determinada área, como conceitos, técnicas e procedimentos utilizados, resultados e contribuições de estudos prévios, além de possibilitar a avaliação da qualidade do conhecimento produzido e identificar possíveis lacunas para que novas pesquisas possam contribuir, acrescentando conhecimento ao já estabelecido (BOTELHO; CUNHA; MACEDO, 2011; SOUSA; RIBEIRO, 2009).

Este estudo baseou-se principalmente nas proposições de Kitchenham (2004) e Kitchenham e Charters (2007) para a definição do protocolo de pesquisa e condução da RSL. A metodologia da pesquisa está descrita nas subseções seguintes.

\subsection{Planejamento da revisão: definição do protocolo}

Kitchenham e Charters (2007) apontam que o método PICOC (PETTICREW; ROBERTS, 2006) é eficaz para a orientação da RSL elucidando os aspectos focais do trabalho e orientando a extração dos dados de interesse para o pesquisador, levando em consideração cinco aspectos principais que formam o acrônimo. Estes pontos aplicados a esta RSL estão descritos a seguir:

- Population (População): trabalhos que tratam da aplicação de um método de decisão multicritério no suporte ao processo decisório;

- Intervention (Intervenção): usar de algum método de decisão multicritério no suporte ao processo decisório;

- Comparison (Comparação): não se aplica, pois o uso do método não será comparado a outros métodos de decisão, visando apenas a coleta das informações pretendidas; 
- Outcome (Resultados): relacionar os seguintes aspectos de interesse, a saber: contexto de aplicação - quando, onde, ramo de atuação da organização, principais métodos de decisão multicritérios mais utilizados, etc;

- Context (Contexto): são trabalhos que destacam a utilização de um método de decisão multicritério no suporte ao processo decisório.

Dentro do protocolo e aliado ao PICOC, a questão de pesquisa (QP) descreve de maneira sucinta qual o objetivo que a pesquisa pretende alcançar, norteando todo o processo da RSL. Neste sentido, esta RSL teve como QP a seguinte indagação "Como os métodos de decisão multicritério estão sendo aplicados no auxílio à tomada de decisão nas organizações?”, baseada no objetivo geral do trabalho.

Visando o cumprimento deste objetivo, recomenda-se que algumas questões específicas (QE) sejam traçadas para que se descreva em detalhes, quais os pontos a serem operacionalizados e que contribuirão no intuito de que a questão de pesquisa seja contemplada, ou seja, os seus objetivos específicos.

Três metas específicas ou QEs foram determinadas. A primeira levou em consideração a investigação das aplicações mais recentes nas organizações, obtendo uma visão atual do cenário. Para este estudo, um recorte de tempo de cinco anos foi delimitado. A segunda considerou a busca de quais métodos, mencionados na seção 2, são utilizados com mais frequência. Por fim, a terceira pergunta foi desenhada para se agrupar as aplicações conforme o tipo de negócio no qual ocorreu a utilização do método multicritério. Portanto, com base nestas justificativas, os objetivos específicos consistiram em:

- "Qual a frequência de estudos nesta área nos últimos 5 anos (período que compreende 2016-2020)?” (QE1);

- "Quais métodos são aplicados com maior frequência no auxílio à tomada de decisão nas organizações?” (QE2);

- “Quais os setores em que as organizações mais se utilizam destas técnicas?”(QE3).

As bases de dados/bibliotecas digitais selecionadas que serviram como fonte dos artigos para este estudo, foram respectivamente a ACM Digital Library, IEEE Explore, Science Direct (Elsevier) e Web of Science, devido a sua notoriedade, credibilidade no meio acadêmico e relevância na pesquisa científica e nos dos estudos do ramo da Administração.

Para efetuar a consulta nestas bases, uma string de busca padrão foi definida a fim de que os resultados retornados estivessem alinhados com os objetivos pretendidos. A string foi montada com base nas palavras-chave do trabalho, nas questões de pesquisa e no PICOC supracitado, resultando na seguinte estrutura:

\section{((“multi-criteria” $\boldsymbol{O R}$ “multi criteria” $\boldsymbol{O R}$ “multiple criteria” $\boldsymbol{O} \boldsymbol{R}$ “MCDM”)}

\section{("organization" OR "enterprise”)}




\section{AND \\ (“decision-making” OR “decision making”)).}

Visando a garantia de uma qualidade mínima dos estudos a serem analisados e seu alinhamento com as diretrizes da revisão sistemática da literatura, um conjunto de critérios foram definidos, tanto para levantar uma base inicial de materiais, os critérios de inclusão (CI), quanto para filtrar os estudos que de fato farão parte da análise, os critérios de exclusão (CE).

Para este trabalho foram formuladas duas formas de inclusão, (CI1) Artigos de revistas e conferências, revisados por pares, que abordam a aplicação de métodos de decisão multicritério nas organizações e (CI2) Estudos importantes citados pelos autores dos artigos identificados durante o estudo exploratório ou obtidos através da pesquisa bola de neve.

Os seguintes critérios de exclusão foram definidos para triagem dos artigos levantados: (CE1) Estudos duplicados ou com o mesmo conteúdo; (CE2) estudos não publicados entre os anos de 2016 a 2020; (CE3) Estudos indisponíveis para consulta ou download nas bases de origem ou em outros sites; (CE4) Estudos incompletos, com apenas o resumo disponível, banners, etc.; (CE5) Estudos que não estão escritos em inglês (devido as bases serem internacionais); (CE6) Estudos que não responderam à questão de pesquisa (QP); (CE7) Estudos que não atendam aos critérios de qualidade (CQ) definidos. Os critérios de inclusão e exclusão foram baseados em Duarte et al. (2020).

A respeito dos critérios de qualidade que compõem o CE7, estes foram subdivididos em duas categorias. A primeira, composta por 6 questões, compôs o grupo de "itens gerais" formado pelos tópicos que tratavam das questões metodológicas, objetivos, resultados, limitações e contribuições dos estudos. O segundo grupo, os "itens específicos", era formado por 4 questões relacionadas a temática desta revisão sistemática, a pergunta principal de pesquisa e as três QEs elaboradas. Para as respostas de cada CQ, foi atribuída uma nota que compôs uma pontuação de corte para a manutenção ou exclusão do artigo na RSL. Os 10 CQs e suas respectivas notas estão descritos no Quadro 2.

A pontuação obtida no CQ de cada artigo foi calculada conforme as notas obtidas em cada pergunta descrita anteriormente e um sistema de pesos definido pelos autores, conforme a equação da Figura 1, bem como a condição para manutenção ou corte do estudo.

Vale salientar que esta classificação, apesar do nome, não se relaciona a qualidade dos estudos em si, apenas a sua pertinência e contribuições para os objetivos desta revisão sistemática. 
Figura 1 - Fórmula para cálculo do CQ dos artigos.

$$
\mathrm{CQ}=\left[\frac{\sum_{\mathrm{QG}=1}^{6}}{6}+\left(\frac{\sum_{\mathrm{QE}=1}^{4}}{4} \times 3\right)\right]
$$

\begin{tabular}{|c|c|c|}
\hline Qualidade & Pontuação & Ação \\
\hline Alta & $\geq 3,0$ & $\begin{array}{c}\text { O estudo não será excluído } \\
\text { da RSL }\end{array}$ \\
\hline Média & $\geq 1,5 \mathrm{e}<3,0$ & $\begin{array}{c}\text { O estudo não será excluído } \\
\text { da RSL }\end{array}$ \\
\hline Baixa & $<1,5$ & $\begin{array}{c}\text { O estudo será excluído da } \\
\text { RSL }\end{array}$ \\
\hline
\end{tabular}

Fonte: Os autores.

Quadro 2 - Definição dos critérios de qualidade (CQ) e suas pontuações.

\section{ITENS GERAIS}

QG1. Definição do problema e motivação do estudo:

(1.0) há uma descrição explícita do problema e da motivação.

(0.5) há uma descrição geral do problema e/ou da motivação.

(0.0) não há nenhum tipo de descrição nem do problema e nem da motivação.

\section{QG2. Há uma descrição metodológica do estudo:}

(1.0) há uma descrição detalhada do método utilizado.

(0.5) há apenas uma descrição simplificada do método utilizado.

(0.0) não há descrição sobre o método utilizado.

\section{ITENS GERAIS (CONT.)}

QG3. As contribuições do estudo referem-se aos resultados do estudo:

(1.0) há uma correlação explícita entre as contribuições e os resultados.

(0.5) não há correlação entre as contribuições e os resultados.

(0.0) não há descrição de contribuições e/ou resultados.

\section{QG4. Há uma descrição sobre a validação do estudo:}

(1.0) há uma descrição formalizada sobre a validação do estudo.

(0.5) apenas algumas evidências foram fornecidas sobre a validação do estudo.

(0.0) não há nenhum tipo de validação para o estudo. 
QG5. Há apresentação de insights para novos estudos:

(1.0) há uma apresentação explícita de insights e/ou lições aprendidas.

(0.5) há uma apresentação de forma geral de alguns insights.

(0.0) não há nenhuma descrição de insights no estudo.

QG6. Há alguma apresentação sobre os limites que o estudo encontrou ou superou:

(1.0) há uma descrição explícita dos limites encontradas nos estudos.

(0.5) há apenas uma descrição em linhas gerais de alguns limites.

(0.0) não há descrição sobre os limites encontradas no decorrer do estudo.

\section{ITENS ESPECÍFICOS}

QE1. O estudo evidencia o método e as ferramentas utilizadas durante o processo:

(1.0) estes aspectos foram devidamente evidenciados.

(0.5) apenas algumas evidências destes aspectos foram relatadas.

(0.0) não há evidências relatadas sobre estes aspectos.

QE2. Há descrição sobre as motivações que orientaram a escolha do método de decisão multicritério:

(1.0) há uma descrição formal sobre as motivações.

(0.5) há uma descrição informal sobre as motivações.

(0.0) não há nenhuma descrição sobre as métricas motivações.

QE3. Há definição das etapas de aplicação das metodologias na tomada de decisão:

(1.0) há uma definição formal sobre a condução da aplicação.

(0.5) há uma definição informal sobre a condução da aplicação.

(0.0) não há nenhuma definição sobre a condução da aplicação.

QE4. Os resultados obtidos através da aplicação do método de decisão multicritério foram relatados:

(1.0) os resultados foram devidamente relatados.

(0.5) apenas algumas evidências dos resultados foram relatadas.

(0.0) não há relatos sobre os resultados.

Fonte: Os autores, com base em Kitchenham (2004); Duarte et al. (2020).

\subsection{Execução da revisão: extração dos dados}

Após a definição do protocolo de pesquisa foi conduzida a fase de extração dos dados da RSL por meio da avaliação crítica dos estudos, com base nos critérios de inclusão e exclusão definidos para avaliação. Para serem incluídos na análise de dados da revisão, os estudos 
deveriam atender a no mínimo um critério de inclusão e não atender a nenhum dos critérios de exclusão. Esta fase foi realizada em três etapas sequenciais: a exclusão de trabalhos duplicados em duas ou mais bases; uma leitura preliminar das informações básicas do trabalho - título, autor(es), ano de publicação, palavras-chave - e; por fim, a leitura do resumo (abstract), objetivos, métodos e resultados dos artigos (caso fosse necessário, era realizada a leitura do trabalho na íntegra).

Para dar suporte a extração de dados foi utilizado o software StArt (State of the Art through Systematic Review) v.3.0.3 beta, desenvolvido pelo LaPES - Laboratório de Pesquisa em Engenharia de Software - que faz parte do Departamento de Informática da Universidade Federal de São Carlos (UFSCar). A ferramenta auxiliou a construção e utilização de formulários padronizados para catalogação e avalição dos dados, além de monitorar o status da pesquisa.

Quadro 3 - Processo de execução da RSL.

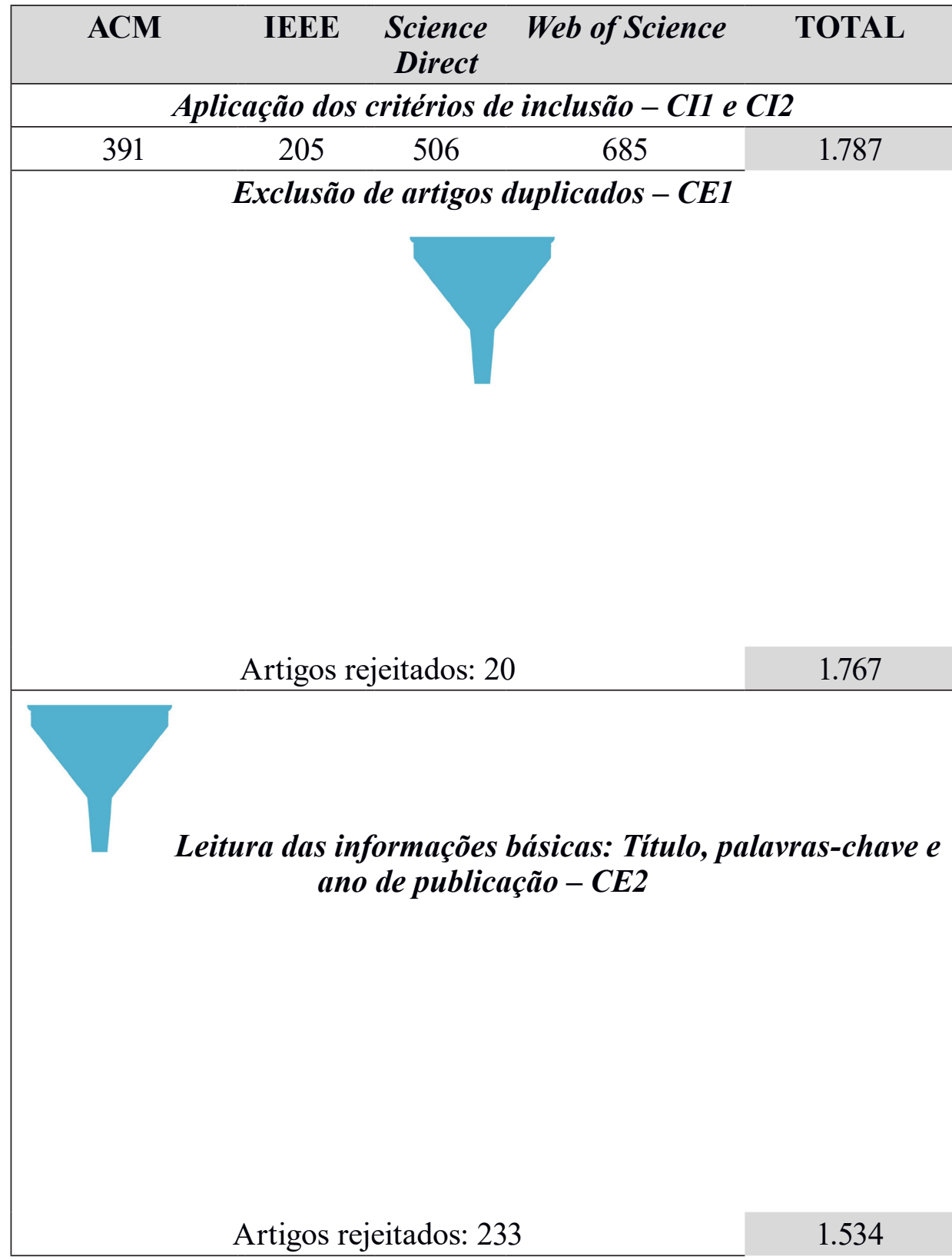




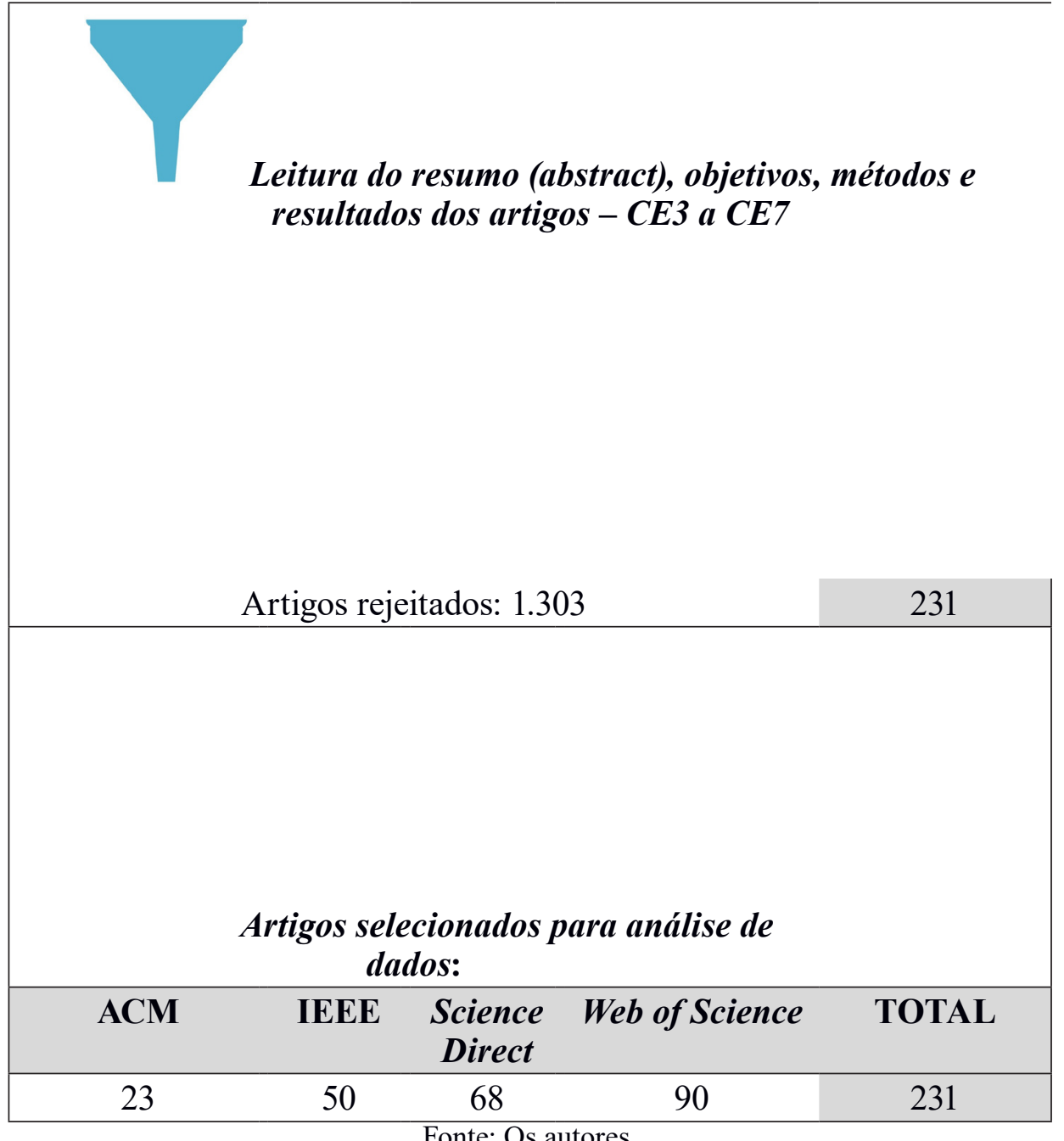

Os procedimentos descritos anteriormente resultaram no retorno de 1.787 estudos das quatro bases selecionadas para esta pesquisa. Após a primeira etapa de extração, 20 artigos foram excluídos por causa de duplicidade em diferentes bases. Durante a segunda etapa de extração, após a leitura das informações básicas dos trabalhos restantes, 233 foram descartados mediante análise dos sete critérios de exclusão definidos. Por fim, depois de leitura mais detalhada dos artigos, 1.303 foram excluídos, resultando no quantitativo final de 231 trabalhos que seguiram para a fase de análise de dados. Este processo está sequenciado no Quadro 3.

\section{Principais resultados}

Esta seção apresenta a análise descritiva do conjunto de estudos selecionados. A Figura 2 demonstra a frequência de artigos aceitos e rejeitados de acordo com as restrições do protocolo de pesquisa, com $13 \%$ dos artigos aceitos. A Figura 3 traz a distribuição percentual dos trabalhos que avançaram para a fase de análise de dados, de acordo com sua base de origem. 
Figura 2 - Quantitativo de estudos após aplicação dos critérios de exclusão (CE).

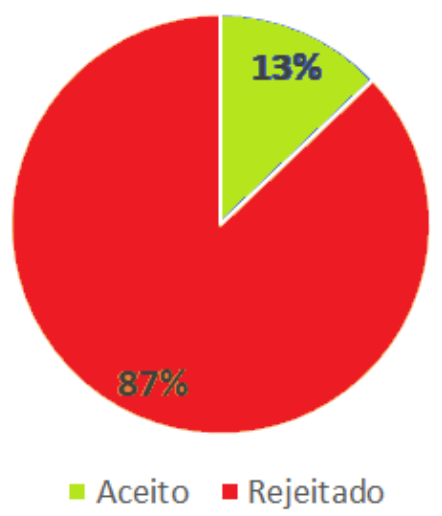

Fonte: Os autores.

Figura 3 - Estudos analisados distribuídos por base de dados.

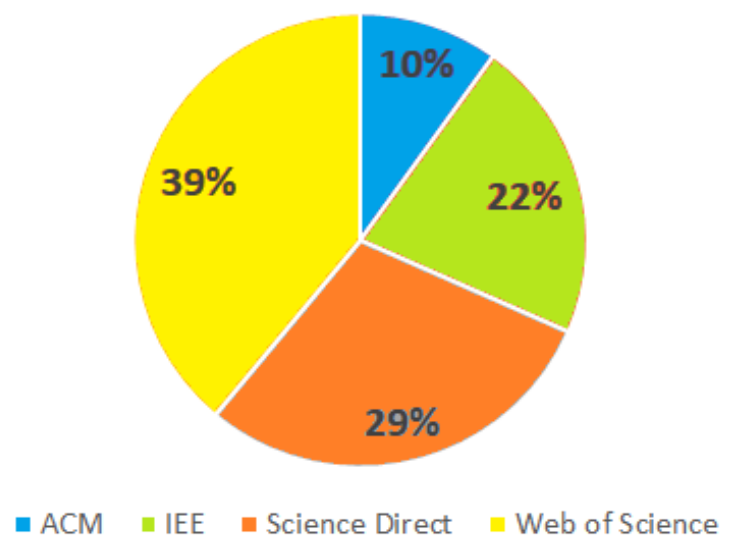

Fonte: Os autores.

Considerando a três questões específicas de pesquisa $(\mathrm{QE})$, a respeito da primeira, $\mathrm{QE} 1$ : "Qual a frequência de estudos nesta área nos últimos 5 anos (período que compreende 20162020)?", a Figura 4 demonstra a quantidade de estudos por ano dentro do recorte de tempo proposto. Através do gráfico, pode-se afirmar que os estudos que tratam de aplicações em organizações dos métodos de decisão multicritério apresentaram uma crescente ao longo do período analisado, demonstrando a sua relevância e maior aplicação dentro da área da administração. 
Figura 4 - Frequência dos estudos selecionados por ano de publicação.

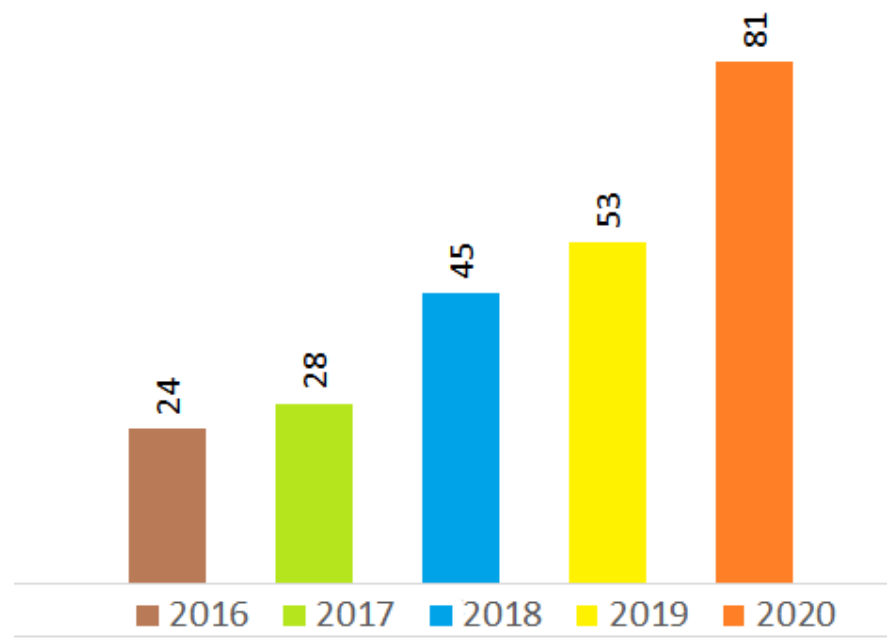

Fonte: Os autores.

Em relação as outras duas questões específicas, QE2: "Quais métodos são aplicados com maior frequência no auxílio à tomada de decisão nas organizações?" e QE3: "Quais os setores em que as organizações mais se utilizam destas técnicas?”, o Quadro 4 sintetiza a resposta para ambas as perguntas.

As organizações encontram-se agrupadas em setores, conforme as similaridades apresentadas nos seus respectivos core business descritos nos trabalhos. Foram oito grupos encontrados: Saúde; Educação; Tecnologia e telecomunicações; Gestão Organizacional (estratégia, recursos humanos, financeiro, logística); Meio ambiente (agricultura, energia e sustentabilidade); Indústria e manufatura; Vendas e serviços (turismo e hotelaria) e Transporte. Dentro desta divisão, as áreas com mais destaque foram Meio Ambiente; Tecnologia e Telecomunicações; e a de Gestão Organizacional.

Neste quadro o somatório dos valores ultrapassa o quantitativo de artigos selecionados para a fase de análise (231), isto porque, em um mesmo trabalho, uma aplicação poderia estar classificada em dois setores simultaneamente, bem como o mesmo estudo poderia abordar a utilização de mais de um método, já que se trata de avaliação de métodos simples, sem combinação.

Os métodos AHP, PROMETHEE (família), TOPSIS, ANP e ELECTRE (família), em suas formas simples, foram os mais presentes, com destaque considerável para os três primeiros. No entanto, uma quantidade de trabalhos apresentou outros tipos em menor frequência como MACBETH, SAW, Fuzzy, SMART, VIKOR, entre outros citados no Quadro 1. Este conjunto foi agrupado na coluna "Outros", com 77 ocorrências.

Apesar das críticas existentes, o AHP foi o método mais utilizado entre os artigos analisados (102 ocorrências). Pereira, Brandalise e Mello (2017) justificam sua amplitude de utilização no apoio à decisão multicritério devido a sua simplicidade e facilidade de operação em comparação a outras técnicas. Os autores ainda destacam que seu funcionamento é similar ao da mente humana no quesito resolução de problemas, atribuindo importância tanto aos dados coletados, quanto ao conhecimento e subjetividade do decisor. 
Quadro 4 - Distribuição das aplicações dos métodos por setores organizacionais.

\begin{tabular}{|c|c|c|c|c|c|c|c|}
\hline & \multicolumn{6}{|c|}{ MÉTODO DE APOIO À TOMADA DE DECISÃO } & \multirow[b]{2}{*}{ TOTAL } \\
\hline $\begin{array}{c}\text { SETOR DE } \\
\text { ATUAÇÃO DA } \\
\text { ORGANIZAÇÃO }\end{array}$ & AHP & ANP & ELECTRE & PROMETHEE & TOPSIS & Outros & \\
\hline Saúde & 10 & 2 & 1 & 8 & 9 & 7 & 37 \\
\hline Educação & 3 & 0 & 0 & 2 & 0 & 2 & 7 \\
\hline $\begin{array}{c}\text { Tecnologia e } \\
\text { telecomunicações }\end{array}$ & 25 & 9 & 2 & 4 & 12 & 13 & 65 \\
\hline $\begin{array}{c}\text { Gestão } \\
\text { Organizacional: } \\
\text { estratégia, recursos } \\
\text { humanos, financeiro, } \\
\text { logística }\end{array}$ & 14 & 8 & 5 & 5 & 11 & 15 & 58 \\
\hline $\begin{array}{l}\text { Meio ambiente: } \\
\text { agricultura, energia e } \\
\text { sustentabilidade }\end{array}$ & 20 & 1 & 0 & 22 & 12 & 13 & 68 \\
\hline $\begin{array}{l}\text { Indústria e } \\
\text { manufatura }\end{array}$ & 15 & 2 & 1 & 7 & 10 & 9 & 44 \\
\hline $\begin{array}{c}\text { Vendas e serviços } \\
\text { (turismo e hotelaria) }\end{array}$ & 1 & 1 & 0 & 3 & 3 & 10 & 18 \\
\hline Transporte & 14 & 0 & 0 & 5 & 4 & 8 & 31 \\
\hline TOTAL & 102 & 23 & 9 & 56 & 61 & 77 & \\
\hline
\end{tabular}

Fonte: Os autores.

Considerando a interseção entre o tipo de método e os setores organizacionais, pode-se apontar três contextos significativos. A aplicação de "AHP no setor de Tecnologia e Telecomunicações" (25), cujas aplicações concentram-se em torno da escolha de sistemas e ferramentas de software foi encontrada, por exemplo, em Petrova (2019) que trouxe em seu trabalho a investigação da ferramenta de ambiente virtual de aprendizagem mais adequada a seus objetivos. De modo similar, Polkowski (2018) trouxe a mesma abordagem para o âmbito das pequenas e médias empresas. Vitorino, Russo e Camanho (2016) utilizaram o AHP para seleção de sistema ERP para um hospital. Além da seleção de ferramental, o método também foi aplicado no desenvolvimento de sistemas (SURBAKTI et al., 2019; ZHICHENG; FENG, 2018) e melhoria, como no caso voltado a segurança de dados pessoais e privacidade de usuários (RIBEIRO; CANEDO, 2020).

A segunda e terceira interseção notória foram a aplicação de "PROMETHEE no setor de Meio Ambiente" (22) e a aplicação de "AHP no setor de Meio Ambiente" (20). Em relação a família PROMETHEE, Safari, Abbaspour e Javid (2020) foram um exemplo de estudo que visou a seleção e priorização dos recursos de irrigação da área verde no Irã; Gokcekus, Ozsahin e Mustapha (2020) analisaram dispositivos de esterilização de água e; Belgin e Balkan (2020) utilizaram o PROMETHEE II para classificar o desempenho ambiental dos setor de manufatura baseado em cinco critérios. Wei et al. (2016) propuseram uma avaliação do sistema de armazenamento de energia utilizando-se da ferramenta. 
A utilização do AHP no setor é principalmente caracterizada pela seleção de fornecedores e locais, conforme Cabuk (2018), na escolha de fornecedores em uma empresa de móveis que opera na região de Mármara, na Turquia e Sari et al. (2020), na definição de locais adequados para a atividade apiária. Os impactos nos setores econômicos, social e ambiental constituíram base para os critérios de Jurik et al. (2020) na triagem de projetos pautados nos critérios do desenvolvimento sustentável. Bayram e Üçüncü (2016) utilizaram o AHP para mapear a situação da indústria de produtos florestais de Taşköprü, na Turquia.

O Quadro 4 evidencia essa complexidade das decisões quando se trata do setor de Meio Ambiente, que obteve o maior número de ocorrência (68), devido a sua multiplicidade de atores de decisão, avaliações de cenário, inter-relacionamento de ações de controle e gerenciamento em esferas civil e pública, modelagem das ações nos diferentes ecossistemas de fauna e flora, entre outras variáveis, conforme os exemplos supracitados.

\section{Conclusões}

Esta Revisão Sistemática da Literatura apresentou a utilização dos métodos de decisão multicritério no processo decisório, por meio da identificação da aplicação de diversas técnicas de MCDM/A, Multicriteria Decision Making e Multicriteria Decision Aid, com base no seu setor de atuação, encontradas na literatura. A RSL demonstrou que nos últimos anos o número de trabalhos vem aumentando gradativamente, o que reforça a importância do tema dentro das competências do administrador e mais especificamente, da figura do tomador de decisão.

Conforme apresentado nos resultados, apesar de existirem casos em que um determinado método foi bastante utilizado em setor específico, o estudo apontou que não há uma uniformidade, as aplicações foram amplamente diversas e adaptadas ao setor de acordo com a necessidade dos decisores, do mercado e cenário em que as empresas presentes nos estudos de caso estão inseridas.

A RSL utilizada para evidenciar os trabalhos apoiados e desenvolvidos pela abordagem multicritério, oferece diferenciais importantes em momentos de complexidade e delicados, nos quais as decisões devem ser apoiadas de tal forma a minimizar as chances de erro uma vez que, esses métodos representam graficamente a decisão e o impacto dela nas demais alternativas e cenários mercadológicos, facilitando muito a visualização do equilíbrio matemático ou trade-off entre elas, como um processo de negócio que envolve múltiplos critérios de escolha, incertezas e que precisa ser apoiado com as práticas existentes de tomada de decisão.

Para Edwards e Barron (1994) a modelagem simples e adequada para solução de problemas, pode diminuir o erro de elicitação com o decisor. Estes modelos fazem com que o processo da tomada de decisão possa ser mais racional possível. Na pesquisa realizada, foram encontrados vários exemplos de estruturação racional de decisão em problemas, categorizadas em oito grupos diferentes.

As limitações do trabalho relacionam-se, sobretudo, com os critérios de inclusão e exclusão utilizados na revisão uma vez que, não considerou os casos que estavam fora do recorte de tempo determinado, das bibliotecas digitais escolhidas, dentre outras condições descritas. Outra limitação diz respeito a linguagem dos estudos analisados, restringida ao idioma inglês. Alguns estudos nacionais foram incluídos na análise, no entanto, muito do que seguiu para as fases finais do estudo, faz parte de um contexto mais internacional, que traz características 
diferentes da realidade das organizações brasileiras.

Deste modo, espera-se que este trabalho tenha contribuído para sistematizar a atividade de coleta, análise das informações obtidas na Revisão Sistemática da Literatura, ao considerar o tratamento de informações e definição de protocolo visando a garantia de uma qualidade mínima dos estudos a serem analisados. Assim, foi utilizado um conjunto de critérios para levantar uma base inicial de materiais, que foram os critérios de inclusão (CI) e; para filtrar os estudos utilizados por este trabalho, que são os critérios de exclusão (CE).

Portanto, pode-se perceber o destaque no uso de métodos MDCM/A na análise de vários casos de tomada de decisão. Algumas oportunidades de pesquisas futuras podem ser levantadas como estudos que retratam o detalhamento das condições e motivações características que envolvem a escolha de determinado método para determinado setor mediante outras revisões sistemáticas; a comparação de métodos dentro do mesmo setor de atuação; aplicação de estudo semelhante no contexto nacional ou regional; além da investigação da aplicação em setores pouco explorados ou não presentes neste estudo.

\section{Referências}

ALMEIDA, A. T. de. Um modelo de decisão para priorização no planejamento de sistemas de informação. Revista Produção, v. 8, n. 2, p. 169-185, 1999.

ALMOGHATHAWI, Y. et al. A multi-criteria decision analysis approach for importance identification and ranking of network components. Reliability Engineering \& System Safety, v. 158, p. 142-151, fev. 2017.

BANA E COSTA, C. A; VANSNICK, J. C. MACBETH - An interactive path towards the construction of cardinal value functions. International Transactions in Operational Research, v. 1, n. 4, p. 489-500, 1994.

BAYRAM, B. Ç.; ÜÇÜNCÜ, T. A case study: assessing the current situation of forest products industry in taŞköprü through swot analysis and analytic hierarchy process. Kastamonu Üniversitesi Orman Fakültesi Dergisi, v. 16, n. 2, p. 510-514, dez. 2016.

BELGIN, O.; BALKAN, D. Environmental performance assessment of manufacturing sectors. Clean Technologies And Environmental Policy, v. 22, n. 6, p. 1405-1415, jun. 2020.

BELLMAN, R. E.; ZADEH, L. A. Decision-Making in a Fuzzy Environment. Management Science, v. 17, n. 4, p. 141-164, dez. 1970.

BOTELHO, L. L. R.; CUNHA, C. C. A.; MACEDO, M. O método da revisão integrativa nos estudos organizacionais. Gestão e Sociedade, Belo Horizonte, v. 5, n. 11, p. 121-136, maio/ago. 2011.

BRANS, J. P.; VINCKE, P. A preference ranking organization method (The PROMETHEE method for multiple criteria decision-making). Management Science, v. 31, p. 647- 656, 1985. 
BRANS, J. P.; VINCKE, P.; MARESCHAL, B. How to select and how to rank projects: The PROMETHEE method. European journal of operational research, v. 24, n. 2, p. 228-238, 1986.

CABUK, Y. et al. The use of analytical hierarchy method in factory establishment placement to prevent environmental pollution in furniture industry. Fresenius Environmental Bulletin, v. 27, n. 2, p. 1191-1195. 2018.

CARVALHO, A. S.; OLIVEIRA, F. B.; RIBEIRO, E. A. Aspectos relevantes na confecção de uma revisão sistemática e metanálise. Evidência, Araxá, v. 7, n. 7, p. 229-236, 2011.

CHARNES, A.; COOPER, W. W.; RHODES, E. Measuring the efficiency of decision making units. European Journal of Operational Research, v. 2, n. 6, p. 429-444, 1978.

DENG, J. L. Introduction to Grey System Theory. Journal of Grey System, v. 1, n. 1, p. 1-24. 1989.

DUARTE, R. B. et al. A systematic literature review on the usage of eye-tracking in understanding process models. Business Process Management Journal, p. 1-22, out/2020.

EDWARDS, W.; BARRON, F. H. SMARTS and SMARTER: Improved simple methods for multiattribute utility measurement. Organizational Behaviour and Human Decision Processes, v. 60, p. 306-325, 1994.

GALVÃO, T. F.; PEREIRA, M. G. Revisões sistemáticas da literatura: passos para sua elaboração. Epidemiol. Serv. Saúde, Brasília, v. 23, n. 1, p. 183-184, jan./mar. 2014.

GIL, A. C. Métodos e técnicas de pesquisa social. 5. ed. São Paulo: Atlas, 1999.

GOKCEKUS, H.; OZSAHIN, D. U.; MUSTAPHA, M. T. Simulation and evaluation of water sterilization devices. Desalination And Water Treatment, v. 177, p. 431-436, 2020.

GUARNIERI, P. Síntese dos Principais Critérios, Métodos e Subproblemas da Seleção de Fornecedores Multicritério. Revista de Administração Contemporânea, v. 19, n. 1, p. 1-25, fev. 2015.

HAIR JR., J. F.; ANDERSON, R. E.; TATHAM, R. L.; BLACK, W. C. Análise multivariada de dados. 6. ed. Porto Alegre: Bookman, 2009.

HAYKIN, S. Redes Neurais: Princípios e Práticas. 2. ed. São Paulo: Bookman, 2001.

HWANG, C. L., YOON, K. Multiple attribute decision making methods and applications. New York: Springer-Verlag, 1981.

JERÔNIMO, T. B. Modelo Multicritério para Classificação Gerencial das Alternativas Estratégicas nas Pequenas e Médias Empresas de Tecnologia da Informação. 2013. 135f. 
Tese (Doutorado em Engenharia de Produção) - Departamento de Engenharia de Produção, Universidade Federal de Pernambuco, Recife.

JURÍK, L.; HORňÁKOVÁ, N.; lANTAVÁ, E.; CAGÁňOVÁ, D.; SABLIK, J. Application of AHP method for project selection in the context of sustainable development. Wireless Networks, p. 1-10, abr. 2020.

KEENEY, R. L.; RAIFFA, H. Decision with Multiple Objectives: Preference and Value Tradeoffs. New York: Wiley, 1976.

KITCHENHAM, B. Procedures for performing systematic reviews, Technical Report TR/ SE-0401. Department of Computer Science, Keele University, Keele, Staffs, UK, 2004.

KITCHENHAM, B.; CHARTERS, S. Guidelines for performing Systematic Literature Reviews in Software Engineering. Technical Report EBSE 2007-001, Keele University and Durham University Joint Report, 2007.

LACOMBE, F. Teoria geral da administração. São Paulo: Saraiva, 2009.

LIMA JUNIOR, F. R.; OSIRO, L.; CARPINETTI, L. C. R. Métodos de decisão multicritério para seleção de fornecedores: um panorama do estado da arte. Gest. Prod., São Carlos, v. 20, n. 4, p. 781-801, 2013.

PAWLAK Z. Rough sets. International Journal of Computer and Information Sciences, v. 11, n. 5, p. 341-356, 1982.

PEREIRA, A. S. A.; BRANDALISE, N.; MELlO, L. C. B de B. Aplicação do método AHP na seleção de terrenos para edificações comerciais na cidade do Rio de Janeiro. Sistemas \& Gestão, v. 11, n. 4, p. 410-422, maio 2017.

PETROVA, V. Using the Analytic Hierarchy Process for LMS selection. Proceedings Of The 20Th International Conference On Computer Systems And Technologies, p. 332-336, jun. 2019.

PETTICREW, M.; ROBERTS, H. Systematic reviews in the social sciences: A practical guide. Malden, MA: Blackwell, 2006.

POLKOWSKI, Z. The Method of Implementation of Information and Telecommunication Technologies In Small And Medium Enterprises. 2018 10Th International Conference On Electronics, Computers And Artificial Intelligence (Ecai), p. 1-6, jun. 2018.

RAIFFA, H. Teoria da decisão: aulas introdutórias sobre escolhas em condições de incerteza. Petrópolis: Vozes, 1977.

RIBEIRO, R. C.; CANEDO, E. D. Using MCDA for Selecting Criteria of LGPD Compliant Personal Data Security. The 21St Annual International Conference On Digital Government 
Research, p. 175-184, jun. 2020.

ROY, B. Classement et choix en presence de points de vue multiples (la methode ELECTRE). Revue française d'automatique, d'informatique et de recherche opérationnelle. Recherche opérationnelle, v. 2, n. 1, p. 57-75, 1968.

ROY, B.; BERTIER, P. La méthode ELECTRE II. Paris: SEMA-METRA, 1971.

SAATY, T. L. Decision Making with Dependence and Feedback: The Analytic Network Process. Pittsburg: RWS, 1996

SAATY, T. L. The Analytic Hierarchy Process. New York: McGraw-Hill, 1980.

SAFARI, A.; ABBASPOUR, M.; JAVID, A. H. The application of multi-criteria (AHPPROMETHEE) decision-making methods in selecting and prioritizing the green area irrigation resources. International Journal Of Environmental Science And Technology, p. 1-12, ago. 2020.

SARI, F.; KANDEMIR, İ.; CEYLAN, D. A.; GÜL, A. Using AHP and PROMETHEE multicriteria decision making methods to define suitable apiary locations. Journal Of Apicultural Research, v. 59, n. 4, p. 546-557, fev. 2020.

SIMATUPANG, T. M.; WRIGHT, A. C.; SRIDHARAN, R. Applying the theory of constraints to supply chain collaboration. Supply Chain Management: An International Journal, v. 9, n. 1, p. 57-70, fev. 2004.

SLOWINSKI, R.; GRECO, S.; MATARAZZO, B. Rough set and rule-based multicriteria decision aiding. Pesquisa Operacional, v. 32, n. 2, p. 213-270, ago. 2012.

SOUSA, M. R.; RIBEIRO, A. L. P. Systematic Review and Meta-Analysis of Diagnostic and Prognostic Studies: A Tutorial. Arquivo Brasileiro de Cardiologia, v. 92, n.3, p. 241-251, 2009.

SURBAKTI, E. E.; PURWANDARI, B.; SOLICHAH, I.; KUMARALALITA, L. Analysis of software development method selection. Proceedings Of The 3rd International Conference On Business And Information Management - Icbim '19, p. 168-173, 2019.

TAN, P.; STEINBACH, M.; KUMAR, V. Introdução ao Data Mining: Mineração de Dados. Rio de Janeiro: Editora Ciência Moderna Ltda, 2009.

VANDERBEI, R. J. Linear Programming: Foundations and Extensions. Kluwer Academic Publishers, 1996.

VITORINO, S.; RUSSO, R. de F. S. M.; CAMANHO, R. Seleção de um sistema de gestão hospitalar por um método multicritério. Iberoamerican Journal Of Project Management, v. 7, n. 2, p. 1-17, dez. 2016. 
WEI, L.; HOU, J.; QIN, T.; YUAN, Z.; YAN, Y. Evaluation of grid energy storage system based on AHP-PROMETHEE-GAIA. 2016 35Th Chinese Control Conference (Ccc), p. 9787-9792, jul. 2016.

ZENELY, M. Linear Multiobjective Programming. New York: Springer-Verlag, 1974.

ZHANG, J.; WU, D.; OLSON, D. L. The method of grey related analysis to multiple attribute decision making problems with interval numbers. Mathematical and Computer Modelling, v. 42, n. 9-10, p. 991-998, nov. 2005.

ZHICHENG, D.; FENG, L. Evaluation of the Smart Campus Information Portal. Proceedings Of The 2018 2Nd International Conference On Education And E-Learning, p. 73-79, 5 nov. 2018. 